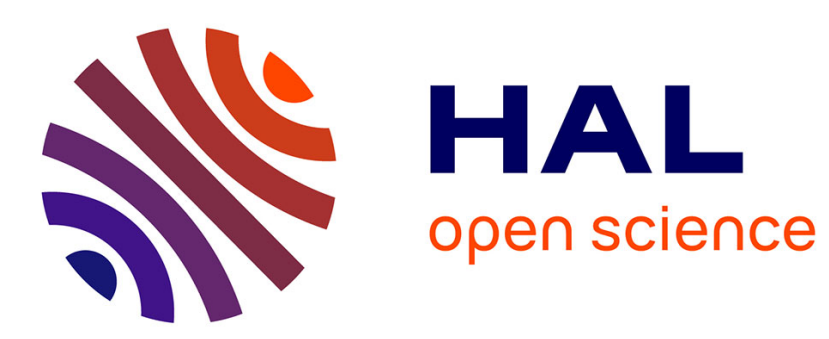

\title{
Voter en Inde: symboles électoraux, système de partis et vote non individuel
}

Christophe Jaffrelot

\section{To cite this version:}

Christophe Jaffrelot. Voter en Inde: symboles électoraux, système de partis et vote non individuel. Revue Française de Science Politique, 1993, 43 (2), pp.301-316. 10.3406/rfsp.1993.394736 . hal$01009734 \mathrm{v} 2$

\section{HAL Id: hal-01009734 \\ https://hal-sciencespo.archives-ouvertes.fr/hal-01009734v2}

Submitted on 23 Aug 2018

HAL is a multi-disciplinary open access archive for the deposit and dissemination of scientific research documents, whether they are published or not. The documents may come from teaching and research institutions in France or abroad, or from public or private research centers.
L'archive ouverte pluridisciplinaire HAL, est destinée au dépôt et à la diffusion de documents scientifiques de niveau recherche, publiés ou non, émanant des établissements d'enseignement et de recherche français ou étrangers, des laboratoires publics ou privés.

\section{(ㅇ)(1) $\$$}

Distributed under a Creative Commons Attribution - NonCommercial - NoDerivatives| 4.0 


\section{Voter en Inde : symboles électoraux, système de partis et vote} non individuel

In: Revue française de science politique, 43e année, n², 1993. pp. 301-316.

\section{Citer ce document / Cite this document :}

Jaffrelot Christophe. Voter en Inde : symboles électoraux, système de partis et vote non individuel. In: Revue française de science politique, 43e année, $n^{\circ} 2$, 1993. pp. 301-316.

http://www.persee.fr/web/revues/home/prescript/article/rfsp_0035-2950_1993_num_43_2_394736 


\title{
Résumé
}

Le taux élevé d'analphabétisme en Inde a conduit cette démocratie (qui a organisé dix élections générales depuis l'indépendance) à élaborer une procédure électorale reposant sur l'usage de symboles : l'acte de vote consiste à cocher dans l'isoloir le symbole correspondant au candidat et à la formation politique de son choix. Ce dispositif a eu des effets induits importants puisque l'attribution définitive d'un symbole aux partis réunissant un certain nombre de voix au niveau national ou régional a contribué à institutionnaliser le système des partis. Au-delà du recours aux symboles, la commission électorale s'est efforcée d'entourer l'acte de vote du plus grand nombre de garanties possibles pour assurer le secret du vote et le préserver des influences extérieures, de manière à réunir les conditions d'un vote rationnel et individuel. La sophistication des mesures prises paraît toutefois en décalage, d'une part, avec les formes concrètes de l'exercice du suffrage, les électeurs tendant à sélectionner leur candidat de façon collective (au niveau de la communauté religieuse ou de la sous-caste), d'autre part avec le vrai défi que constitue la montée des violences autour des bureaux de vote.

\begin{abstract}
Voting in India : electoral symbols, party system and non-individu al voting

The high rate of illiteracy in India has led that democracy (which has had ten legislative elections since independence) to work out an électoral procedure based on the use of symbols : the voting act consists in checking on the ballot the symbol corresponding to the candidate and the political group of one's choice in the voting booth. This System has had many secondary effects, since the definitive attribution of symbols to parties obtaining a certain number of votes on the national or regional level has contributed to the institutionalization of the party system. Beyond the use of symbols, the Election Commission has endeavored to surround the voting act with the maximum of possible guarantees in order to ensure secrecy and to preserve it from outside influences so as to fulfil the conditions for rational and individual voting. The sophistication of the measures taken seems however, on one hand, to lag behind the concrete forms of exercising the suffrage, as the voters tend to select their candidates collectively (at the religions community or the subcaste level); on the other hand, it lags behind the real challenge, that of the rise of violence around voting places.
\end{abstract}

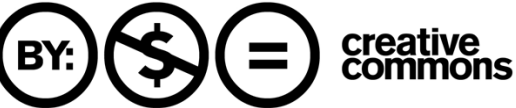




\section{VOTER EN INDE \\ SYMBOLES ÉLECTORAUX, SYSTEMME DE PARTIS \\ ET VOTE NON INDIVIDUEL}

\section{CHRISTOPHE JAFFRELOT}

\section{$\mathrm{L}$}

A République Indienne est souvent présentée comme «la plus grande démocratie du monde ", en raison surtout de sa capacité à organiser des élections libres à intervalles réguliers. Depuis 1951, dix scrutins ont ainsi eu lieu pour renouveler la Chambre basse de New Delhi, la Lok Sabha. Par deux fois (en 1977 et 1989), ces consultations se sont traduites par une alternance politique aux dépens du parti du Congrès.

Les conditions socio-culturelles et démographiques du pays compliquent pourtant le processus électoral : le corps électoral est passé de 173,2 millions d'électeurs en 1952 à presque 400 millions en 1984, croissance d'autant plus importante que le taux de participation, sur la même période, a progressé de 45,7 à $64,1 \%$. En 1989 , l'octroi du droit de vote aux jeunes de 18 à 21 ans a porté le corps électoral à près de 499 millions de personnes, et 298,3 millions d'Indiens l'ont exercé. Les difficultés liées à la masse de l'électorat sont en outre aggravées du fait de son analphabétisme : en 1990, presque $52 \%$ de la population étaient encore illettrés.

L'Inde s'est efforcée de relever le double défi du nombre et de la sous-éducation, d'une part, en déployant d'énormes moyens matériels à l'occasion de chaque élection (plus de 1,5 million d'urnes sont ainsi déplacées à travers le pays pour que le scrutin se déroule sur deux journées dans 595000 bureaux de vote) et, d'autre part, en élaborant une procédure électorale qui permette aux citoyens de s'exprimer dans de bonnes conditions. L'acte de vote s'est ici trouvé associé à la reconnaissance de symboles électoraux dont le rôle croissant a contribué à institutionnaliser le système des partis.

Au-delà du recours aux symboles, la commission électorale, une instance administrative indépendante en charge du processus électoral, s'est efforcée d'entourer l'acte de vote du plus grand nombre de garanties possibles (en termes de secret du vote et de lutte contre les influences extérieures) de manière à réunir les conditions d'un vote rationnel et individuel. La sophistication des mesures prises dans cette perspective paraît toutefois en décalage, d'une part, avec les formes concrètes du suffrage (les électeurs tendant à sélectionner leur candidat à partir de critères communautaires ou même de façon collective), d'autre part avec le vrai défi que constitue la montée des violences autour des bureaux de vote. 


\section{LE RECOURS AUX SYMBOLES ÉLECTORAUX \\ ET LE SYSTEMME DES PARTIS}

L'Inde a hérité sa façon de voter, comme tant d'autres pratiques et institutions politiques, des techniques introduites par les Britanniques. A la fin de l'époque coloniale, un nombre important d'Indiens s'étaient vu accorder le droit de vote lors des réformes constitutionnelles de 1909, 1919 et 1935 (date à laquelle 30 millions d'Indiens, soit un sixième de la population adulte mâle, possédaient ce droit). Le pouvoir britannique s'était efforcé de contourner l'obstacle de l'analphabétisme, de plus en plus sérieux à mesure que la franchise s'abaissait, en instituant deux types de procédures selon les régions : ou les officiels en charge du bureau de vote "aidaient" les électeurs hésitants à inscrire le nom des candidats sur leur bulletin de vote (ce qui rendait caduque la clause légale du secret), ou les urnes étaient peintes d'une couleur particulière pour chaque candidat, et l'électeur pouvait l'identifier même s'il n'arrivait pas à lire son nom ${ }^{1}$.

L'Inde indépendante s'est efforcée d'améliorer le second système en recourant à des symboles électoraux, d'abord inscrits sur les urnes puis sur des bulletins de vote.

\section{LE DISPOSITIF EN PLACE DANS LES ANNÉES 1950: LE BALOTTING SYSTEM}

Les élections de 1951-1952 et celles de 1957 furent organisées suivant une procédure dite balotting system, qui consistait à distinguer les urnes non plus par leur couleur, mais par l'inscription des symboles associés aux candidats. La commission électorale publia en 1951 une liste de symboles parmi lesquels chaque candidat d'une circonscription devait en choisir trois par ordre de préférence. Le représentant local de la commission électorale, après avoir confronté toutes les demandes, attribuait un symbole à chaque candidat pour qu'il puisse le faire connaître au cours de sa campagne électorale ${ }^{2}$. Dans tous les bureaux de vote d'une même circonscription, on trouvait une salle fermée par un rideau, dans laquelle étaient alignées les urnes des différents candidats. Les électeurs se munissaient, à l'entrée du bureau de vote, de bulletins tous identiques et les déposaient dans l'urne de leur choix.

Il y avait en fait deux types de bulletin - des verts et des roses parce que les élections à la Chambre basse de New Delhi et celles pour les Assemblées législatives des Etats fédérés avaient lieu au même moment. Chaque électeur avait donc deux bulletins à disposer dans deux urnes différentes, au minimum.

1. D. Butler, A. Lahiri, P. Roy, India decides. Elections 1952-1989, New Delhi, Living Media, 1989, p. 15.

2. B. Graham, "Electoral symbols and party identification in Indian politics", dans P. Lyon, J. Manor (eds), Transfer and transformation. Political institutions in the new Commonwealth, Leicester, Leicester University Press, 1983, p. 72. 
Dans certaines circonscriptions, la situation était plus complexe encore, car certains sièges étaient réservés aux intouchables ou aux tribus. La Constitution de 1950 prévoit en effet qu'un quota de sièges des assemblées électives soit réservé à ces populations défavorisées. Ces quotas doivent être proportionnels au pourcentage que ces communautés représentent au niveau national en ce qui concerne le Parlement central, et au niveau des Etats fédérés pour ce qui est des Assemblées législatives. Dans certaines circonscriptions dites doubles (ou triples), où ces populations sont particulièrement nombreuses, les électeurs devaient donc élire un candidat sans qualification, un intouchable et/ou un aborigène. Chaque parti pouvait présenter un candidat pour tous ces sièges, sans compter les indépendants. Les urnes de ces candidats au(x) siège(s) réservé(s) étaient marquées du même symbole que ceux des autres candidats du même parti, mais il était cerclé de noir ${ }^{1}$.

En raison, d'une part, de la tenue simultanée des élections aux niveau national et dans les Etats, et, d'autre part, du système des circonscriptions doubles ou triples, une procédure électorale, qui semblait avoir été assez simplifiée pour permettre aux électeurs analphabètes de s'exprimer, se révélait finalement trop complexe. Le nombre des urnes présentes dans un même bureau de vote était source de méprises : les électeurs confondaient celles des candidats au Parlement et aux Assemblées législatives, ou glissaient leurs deux bulletins dans la même urne. Le même phénomène se produisait avec les urnes des candidats intouchables ou aborigènes $^{2}$. En 1957, dans une circonscription du Bengale occidental, 133063 bulletins sur 990800 furent déclarés nuls parce qu'ils avaient été glissés dans la mauvaise urne ${ }^{3}$.

Ce système électoral présentait deux autres inconvénients. D'une part, les candidats pouvaient accuser les officiels en charge du bureau de vote de transférer certains bulletins d'une urne dans l'autre, après la clôture du scrutin, parce qu'ils ne comportaient aucun signe distinctif. D'autre part, le secret du vote n'était pas absolument garanti : le rideau derrière lequel étaient disposées les urnes pouvait ne pas masquer tout à fait les déplacements de l'électeur devant la rangée des urnes. Cette procédure fut donc remplacée par une autre au début des années 1960.

\section{LE MARKING SYSTEM}

Le marking system fut utilisé pour la première fois en 1960 à l'occasion d'élections partielles, puis de façon régulière à partir des élections générales de 1962. Les urnes ornées de symboles avaient disparu. Les symboles se trouvaient désormais sur les bulletins de vote, en face du nom des candidats, pour aider les électeurs qui n'auraient pas pu le déchiffrer. Il y avait un bulletin pour les élections au Parlement et un

1. Election Commission, Report on the second general elections in India, 1957, New Delhi, 1959, p. 92.

2. Ibid.

3. Ibid., p. 164. 


\section{Christophe Jaffrelot}

autre pour les élections à l'Assemblée législative, où le nom de tous les candidats avec leur symbole était imprimé (annexe 1). L'acte de vote se déroulait désormais de la manière suivante ${ }^{1}$ :

- Dans un bureau de vote, le premier agent officiel repérait le nom de l'électeur sur les listes électorales, actualisées chaque année, et inscrivait dans un registre le numéro du bulletin de vote pour les élections à l'Assemblée de l'Etat avant de le passer à un collègue.

- Celui-ci faisait de même avec le bulletin de vote pour les élections au Parlement avant de transmettre le tout à un troisième officiel.

- Cet officiel communiquait les deux bulletins à l'électeur et lui marquait l'index de la main gauche à l'encre indélébile pour le repérer sans mal s'il cherchait à voter une seconde fois.

- Un quatrième officiel reprenait ensuite les bulletins de vote à l'électeur et lui expliquait comment il fallait pratiquer, en apposant un tampon encreur, qu'il lui confiait, en face du symbole et du nom de son choix. Il lui rendait le bulletin de vote des candidats à l'Assemblée législative et passait celui concernant le Parlement à un cinquième collègue. L'électeur entrait dans l'isoloir pour appliquer le tampon en secret.

- Il récupérait ensuite le second bulletin et faisait de même avant de glisser les deux bulletins dans l'urne qui était désormais unique ${ }^{2}$.

Cette façon de voter, codifiée par la commission électorale ${ }^{3}$, changera peu, sauf qu'à partir de 1971-1972 les élections au Parlement et celles aux Assemblées législatives ne se dérouleront plus en même temps. Le marking system avait permis de simplifier l'acte de vote, objectif primordial de la commission électorale.

Le recours à des symboles électoraux pour surmonter l'obstacle de l'analphabétisme eut de grandes répercussions sur le système politique dans la mesure où il contribua fortement à institutionnaliser les partis.

\section{LES SYMBOLES ÉLECTORAUX}

ET L'IDENTIFICATION DES PARTIS POLITIQUES

L'adoption du système des symboles électoraux conduisit les partis à revendiquer l'attribution d'un symbole qui leur soit propre. La commission électorale elle-même était désireuse de voir tous les candidats d'un même parti s'identifier à un seul symbole pour simplifier sa tâche. En 1951, quatorze partis furent reconnus comme ayant droit à un symbole propre. L'état-major de chacun de ces partis était donc requis de communiquer à la commission électorale le nom de ses candidats

1. Pour une reconstitution moins abstraite des modalités de cet acte de vote, voir W. Hauser, W. Singer, "The democratic rite. Celebration and participation in the Indian elections », Asian Survey, 26 (9), septembre 1986, p. 951-956.

2. Les circonscriptions doubles ou triples avaient en effet disparu. Il n'y avait plus qu'un élu par circonscription, un certain nombre de ces circonscriptions territoriales étant réservées aux intouchables et aux aborigènes.

3. La reconstitution qui précède provient des directives de la commission électorale (Election Commission, Report on the third general elections in India, 1962, vol. 1, General, New Delhi, 1965, p. 66-67). 
auxquels on pouvait affecter le symbole du parti. Comme l'écrit Bruce Graham :

«De facto, la Commission avait accepté la mention du nom d'un candidat sur la liste d'un parti comme une preuve suffisante de son affiliation, sans attacher beaucoup d'importance à d'autres critères d'appartenance politique tels que les buts et les déclarations du candidat, l'appui qu'il recevait d'associations informelles et des branches locales de partis politiques ou encore son appartenance aux groupes parlementaires des assemblées sortantes $»^{1}$.

Cette procédure, ajoute B. Graham, « renforça considérablement le pouvoir de recrutement des états-majors des partis politiques ». Ce phénomène s'accentua encore lorsqu'en 1953 la commission électorale décida de ne reconnaître le droit à un symbole, au niveau national, qu'aux partis ayant obtenu plus de $3 \%$ des suffrages exprimés aux élections parlementaires. Seuls quatre partis satisfaisaient à ce critère. II existait cependant une liste séparée pour les partis ayant atteint le même seuil aux élections des Assemblées législatives, compétition pour laquelle ils gardaient le droit à leur symbole (annexe 2).

Tous les partis qui se situaient en deçà de ces seuils perdirent un moyen privilégié d'affirmer leur existence, de faire connaître leur identité : leurs candidats se voyaient attribuer les symboles électoraux qui n'étaient pas réservés aux partis reconnus et surtout leurs symboles pouvaient changer d'une élection à l'autre.

Cette procédure servait ainsi les grands partis dont l'identité, incarnée par des symboles stables à travers toute l'Inde, s'affirmait; d'autant qu'en 1968 les seuils furent portés de 3 à $4 \%$. Le chef de la commission électorale justifia en partie le système des symboles et ces nouveaux seuils en déclarant qu'ils permettraient de réduire le nombre des partis ${ }^{2}$.

Les grandes formations furent rapidement associées à leur symbole, car ils en assuraient sans cesse la promotion. D'une part, les slogans y faisaient et $\mathrm{y}$ font référence de manière explicite. Depuis que le Congrès a la main pour symbole, il appelle volontiers l'opinion à lui «donner un coup de main » ou à y «mettre la main », quant au parti nationaliste hindou, le Jana Sangh, dont le symbole a longtemps été la lampe, il invitait les électeurs à se laisser guider par elle. D'autre part, les grands partis ont vite utilisé leurs militants pour apprendre aux électeurs à reconnaître leur symbole sur un bulletin de vote. De faux bulletins de vote où seul le symbole du parti est vrai sont ainsi utilisés à des fins pédagogiques (voir, en annexe 3, un faux, utilisé à Bhopal pour les élections de 1989 par les militants du Bharatiya Janata Party, le successeur du Jana Sangh, dont le symbole est la fleur de lotus).

L'importance de l'enjeu que représentaient les symboles électoraux apparut clairement après la première scission du Congrès en 1969. Le groupe qui avait fait sécession contre Indira Gandhi, le Congrès O (pour

1. B. Graham, «Electoral symbols...", dans P. Lyon, J. Manor (eds), Transfer and transformation, op. cit., p. 74.

2. Ibid., p. 78 . 
"Organisation »), demanda à se voir attribuer le symbole du rouet. Le Congrès R (pour «Ruling») protesta que ce symbole était proche du drapeau national (où figure une roue, symbole bouddhiste du dharma). Il réclama pour lui-même le symbole de l'enfant. La commission électorale trancha en n'affectant aux deux partis que leur second choix : la femme tournant le rouet pour le Congrès $O$, et la vache avec son veau pour le Congrès $R$. Les partis d'opposition objectèrent alors que ce symbole avait des connotations religieuses propres à attirer l'électeur hindou, la vache revêtant un caractère sacré dans l'hindouisme ${ }^{1}$.

En 1977, lorsque quatre partis d'opposition (le Congrès $\mathrm{O}$, les socialistes, les nationalistes hindous et le Bharatiya Lok Dal, (BLD, parti paysan)) s'unirent pour former le Janata Party (parti du peuple), ils commencèrent par utiliser le symbole électoral du Congrès $O$ dans le Sud tamoul et celui du BLD - un laboureur figurant dans une roue dans le Nord où il était le plus connu ${ }^{2}$. Quand le Janata Party commença à se désintégrer en 1979, le Janata Party (Secular) revendiqua ce symbole, mais la commission électorale lui attribua celui du paysan labourant son champ ${ }^{3}$.

Un conflit comparable avait opposé, peu avant, les deux factions du Congrès qui avaient formé, en 1978, le Congrès U (pour Urs, son chef) et le Congrès I (pour Indira Gandhi). Tous deux revendiquaient le symbole de la vache et du veau que la commission électorale préféra garder. Le Congrès I se vit finalement attribuer la main et le Congrès $U$, le rouet ${ }^{4}$.

Ces conflits autour des symboles électoraux sont révélateurs de l'importance qu'ils ont acquise pour identifier les partis politiques; d'autant que ceux-ci sont généralement parvenus à obtenir des symboles reflétant leur idéologie, voire leur base sociale, ou à y infuser un tel contenu: pour les partis communistes, par exemple, la faucille apparaît associée aux épis de blé ou au marteau et à l'étoile maoïste. Autre exemple, les partis paysans ont toujours réussi à faire figurer un laboureur sur leur symbole électoral. Certains cas sont toutefois plus subtils.

Le Congrès a tout d'abord utilisé une paire de bœufs pour symbole, ce qui renvoie à la culture de la terre pour laquelle les bovins servent de bêtes de trait, mais aussi au respect que suscite la vache dans la culture hindoue. Ce qui apparaît encore plus nettement dans le symbole de la vache et $\mathrm{du}$ veau. Le rouet, tout aussi prisé par certaines factions congressistes, renvoie, lui, au prestige de Gandhi qui filait volontiers le coton et appelait l'Inde à gagner de cette façon l'autosuffisance. Quant au parti nationaliste hindou, le Jana Sangh, qui s'était vu attribuer le symbole de la lampe en 1951, il sut la présenter comme l'instrument

1. Election Commission, Report on the fifth general elections, India, 1971-72. Narrative and reflective part, New Delhi, 1972, p. 67.

2. Election Commission, Report on the sixth general elections to the Lok Sabha, New Delhi, vol. 1, 1978, p. 103.

3. Election Commission, Report on the general elections to the House of the People,

vol. 1, Narrative, Jaipur, Government Central Press, 1980, p. 43.

4. Ibid., p. 42. 
utilisé dans les temples pour éclairer la divinité et surtout accomplir les prières du matin et du soir.

$\mathrm{Au}$ total, la procédure électorale consistant à associer un symbole à chaque candidat et à réserver leur symbole aux partis a permis de surmonter l'obstacle de l'analphabétisme mais n'aura pas été sans incidence politique; l'institutionnalisation du système des partis en procède largement.

Les efforts de la commission électorale pour élaborer une procédure électorale propre à tourner l'obstacle de la sous-éducation s'inscrivent cependant dans une visée plus large dont le but est d'offrir aux électeurs les conditions d'un vote autonome et raisonné.

\section{L'UTOPIE DE L'ÉLECTEUR INDIEN COMME « ACTEUR RATIONNEL »}

\section{POUR UN ACTE DE VOTE INDIVIDUEL ET RÉFLÉCHI}

La commission électorale s'est toujours souciée d'améliorer les conditions de l'acte de vote pour que celui-ci soit le fait d'une volonté individuelle, conformément au modèle occidental dont s'inspirait la République Indienne. Dans cette visée, le secret du vote, le contrôle de l'identité des électeurs et la lutte contre les influences extérieures furent l'objet d'une attention constante.

\section{Les progrès du vote secret}

Le remplacement du système des urnes par le marking system avait déjà permis d'améliorer le secret du vote étant donné que, dans le cas de la première procédure, il était possible de repérer l'urne devant laquelle s'immobilisait l'électeur pour y glisser le bulletin, en dépit du rideau faisant écran. Le marking system n'en comportait pas moins des lacunes : au moment de remettre son bulletin de vote à l'électeur, l'agent du bureau de vote inscrivait le numéro imprimé sur le bulletin en face du nom de l'électeur dans les registres électoraux; il suffisait ensuite, au moment du dépouillement, de rapprocher ce numéro du bulletin pour connaître le vote exprimé.

A partir des élections de 1971 , on introduisit un système de carnet à souche d'où l'agent du bureau de vote détachait les bulletins. Il inscrivait sur le talon le numéro sous lequel l'électeur était inscrit dans le registre électoral, demandait à l'électeur de signer sur ce talon et soulignait le nom de l'électeur dans le registre pour indiquer qu'un bulletin lui avait été remis. Après le dépouillement, souches et bulletins étaient stockés sous scellés, et seul le tribunal pouvait ordonner la levée de ces derniers pour un éventuel recomptage. Pour retrouver le vote émis par un électeur, il fallait désormais rapprocher trois documents et non plus deux : le bulletin, le talon (deux pièces où figuraient le même numéro de série) et 


\section{Christophe Jaffrelot}

les registres électoraux où figuraient le numéro de l'électeur inscrit sur le talon au moment du vote ${ }^{1}$.

Ces précautions visant à assurer le secret du vote devaient surtout soustraire les électeurs au contrôle d'une autorité extérieure. Cet effort pour émanciper les électeurs de l'influence des dominants locaux s'était cependant traduit, dès les années 1950, par des mesures plus directes.

\section{La lutte contre les influences extérieures}

La façon la plus courante de gagner la faveur des électeurs consistait, pour les candidats, à les véhiculer jusqu'au bureau de vote. Le Representation of the People Act de 1951 stipula donc que «le fait, pour un candidat, son adjoint ou tout autre personne, de louer ou de procurer, contre un paiement ou autrement, n'importe quel véhicule pour le transport d'un électeur (autre que le candidat lui-même, des membres de sa famille ou son adjoint) vers ou depuis un bureau de vote, est une pratique illégale ${ }^{2}$.

Des infractions à cette clause, en dépit des peines encourues par leurs auteurs, ayant été observées, la comission électorale s'employa à en diminuer le risque, en multipliant les bureaux de vote de manière à réduire la distance qu'un électeur moyen avait à parcourir pour aller voter. En 1962, elle était de 3 miles, sauf dans les zones faiblement peuplées; en 1971, elle fut ramenée à 1,25 mile.

Les communautés les plus vulnérables aux influences extérieures étant les intouchables, la commission électorale fit aussi en sorte que les bureaux de vote soient établis dans leurs quartiers (qu'il s'agisse d'une circonscription réservée ou non) pour contrer les mesures d'intimidation éventuelles de la part des hautes castes ${ }^{3}$. En 1980, la commission électorale recommanda d'appliquer cette règle même si le quartier intouchable en question représentait moins de 750 électeurs, la taille jusqu'alors minimale pour un bureau de vote ${ }^{4}$.

Dans la même perspective, il fut décidé en 1971 que les bulletins de vote ne seraient pas dépouillés dans les bureaux de vote mais dans le chef-lieu des cantons (qui correspondaient, grosso modo, aux circonscriptions des Assemblées législatives). La commission électorale expliqua que cette innovation avait " éliminé dans une large mesure les manouvres d'intimidation des électeurs appartenant aux milieux les plus faibles, pauvres et minoritaires ... avant les élections, et leur persécution après celles-ci $\rangle^{5}$.

En effet, le dépouillement au bureau de vote permettait de deviner le vote de telle ou telle communauté massivement présente dans la zone en question. Les candidats malheureux, lorsqu'ils étaient issus de castes

1. Report on the fifth general elections, op. cit., p. 203-205.

2. Cité dans V.C. Viraraghavan, "Election law and procedure», dans R.V. Krishna Ayyar (ed.), All India election guide, Madras, Oriental Publishers, 1956, p. 129.

3. Report on the third general elections, op. cit., p. 35, et Report on the fifth general election, op. cit., p. 201.

4. Report on the general elections to the House of the People, op. cit., p. 70.

5. Report on the fifth general elections, op. cit., p. 203. 
dominantes ou disposaient d'un pouvoir quelconque (économique ou politico-administratif), pouvaient alors user de représailles. Longtemps, ces notables avaient bénéficié de ce qu'on appelle en Inde des " banques de vote ", c'est-à-dire de gisements de voix qui leur étaient acquis en raison de leur influence locale. La remise en cause du dépouillement dans le bureau de vote revenait à supprimer un des moyens de contrôle de leur «banque de vote» par ces notables.

Cet acquis fut toutefois perdu en 1979, sous la pression de tous les partis politiques qui souhaitaient, avant tout, être en mesure d'apprécier l'état de leur implantation locale.

\section{L'identification des électeurs}

Un autre aspect de l'acte de vote que la commission électorale s'est attachée à soustaire aux risques de fraude concerne l'identification des électeurs. Le système mis en place dans les années 1950 consistait à leur marquer l'index de la main gauche de manière à repérer ceux qui tenteraient de revenir voter. D'emblée, cette procédure s'avéra peu fiable ${ }^{1}$.

Après les élections de 1957, il fut donc envisagé d'introduire un système de cartes d'identité qui n'étaient pas obligatoires à cette date. L'expérience fut d'abord tentée à Calcutta où l'administration se chargea d'aller photographier les électeurs à leur domicile de manière à garder un cliché pour les listes électorales et d'en apposer un autre sur les cartes d'identité. L'opération échoua dans trois cas sur huit, les femmes refusant d'être prises en photo et nombre d'électeurs s'avérant introuvables à leur domicile, quelle que soit l'heure. Le coût de l'opération se révéla en outre exorbitant. La seule amélioration enregistrée en 1962 consista à autoriser tout agent d'un bureau de vote à mettre en doute l'identité d'un électeur à condition de déposer 2 roupies auprès du président $\mathrm{du}$ bureau ${ }^{2}$.

Une étape supplémentaire fut franchie lors des élections de 1971 : la commission électorale recommanda, alors, la nomination de femmes pour superviser les bureaux de vote où les femmes voilées risquaient d'être nombreuses (essentiellement dans les quartiers musulmans), ce qui facilitait les contrôles d'identité ${ }^{3}$.

A l'occasion des élections de 1980 , le système des cartes d'identité fut introduit à titre expérimental dans l'Etat du Sikkim. Mais les citoyens s'y montrèrent soupçonneux, et seuls $70 \%$ de la population de ce tout petit Etat furent enregistrés. La commission électorale souhaite toujours généraliser ce système. Pour désamorcér la méfiance des Indiens, elle propose que la même carte serve de carte de sécurité sociale, pour

1. En 1957 , on envisagea de remplacer à l'encre par une vaccination contre la variole dont la trace restait longtemps visible (Report on the second general elections, op. cit., p. 106). Cette suggestion est révélatrice de la confusion des genres qu'autorise la conception officielle de l'acte électoral en Inde : il s'agit d'abord d'une manifestation de civisme, au même titre que la vaccination.

2. Report on the third general election, op. cit., p. 73 .

3. Report on the fifth general election, op. cit., p. 75 . 


\section{Christophe Jaffrelot}

l'obtention des rations alimentaires, l'admission à l'université, l'embauche, l'enregistrement ou la vente de biens immobiliers ${ }^{1}$.

Multiplier ainsi les attraits est révélateur de la défiance suscitée par l'Etat (qui est d'abord vu comme l'agent du fisc dans un pays où l'évasion fiscale est très répandue). Cela confirme surtout la faible différenciation du domaine politique ; l'acte de vote ne semble pas fonder la citoyenneté plus que d'autres registres de la vie sociale, tous ici pointent vers une même dimension, le civisme.

Au total, la commission électorale s'est toujours efforcée de réunir les conditions d'un vote secret, sûr et échappant aux influences extérieures. Son souci de la rigueur, voire son perfectionnisme, s'est même traduit par des mesures ou recommandations très sophistiquées : la trace du tampon appliqué sur les bulletins s'imprimant parfois en face d'un second symbole lorsqu'on les pliait avant que l'encre ne soit sèche, à partir de 1971, on utilisa un tampon dont le dessin figurait des rayons courbés dans le sens des aiguilles d'une montre (ce qui permettait de le distinguer des traces éventuelles) ${ }^{2}$. Autre signe révélateur du même souci de rigueur : les bureaux de vote devaient être installés, dans la mesure du possible, dans des bâtiments publics qui possédaient une cour de 20 mètres carrés au moins ${ }^{3}$. Les bureaux de vote devaient pouvoir ne pas être mixtes de manière à ce qu'une file d'attente soit réservée aux femmes. La vente d'alcool, était-il recommandé, devait être interdite le jour $\mathrm{du}$ vote. La commission électorale multiplie aussi les conseils aux électeurs par voie d'affiches ou dans les journaux.

A ce stade, le fait troublant réside dans le contraste évident entre, d'une part, les efforts de la commission électorale pour garantir un vote raisonné qu'aucun vice de forme ou approximation ne puisse entacher, et, d'autre part, les conditions concrètes de l'acte électoral.

\section{CASTÉISME, COMMUNAUTARISME ET FRAUDES}

\section{Le mythe de l'électeur individuel}

Il n'y a guère que dans les milieux occidentalisés des plus grandes villes de l'Inde que le vote puisse parfois s'analyser comme le produit d'une réflexion purement personnelle. Cette décision est en effet, dans la plupart des cas, surdéterminée par des considérations collectives qui tiennent à l'appartenance communautaire ou de caste.

Les communautés religieuses tendent à se replier de plus en plus sur elles-mêmes au plan électoral. Traditionnellement, les minorités musulmane et chrétienne votaient de préférence pour l'un des leurs, de manière à avoir des représentants au Parlement et dans les Assemblées législatives. Depuis les années 1980 , ce type de comportement s'étend parfois aux hindous qui votent de moins en moins volontiers pour un musulman, voire pour un chrétien.

1. Report on the general elections to the House of the People, op. cit., p. 37.

2. Report on the fifth general election, op. cit., p. 202.

3. Report on the general elections to the House of the People, op. cit., p. 69. 
Le même phénomène se retrouve au niveau des castes. Tous les partis examinent la composition par castes d'une circonscription avant de sélectionner leur candidat. Leur choix se porte généralement sur un leader de la caste dominante, c'est-à-dire la plus nombreuse sans jamais (ou presque) être majoritaire, mais il peut aussi être judicieux d'investir quelqu'un d'une autre caste sur lequel les votes de toutes les castes les moins nombreuses, et parfois hostiles à la caste dominante, sont susceptibles de se porter.

Ces stratagèmes renvoient pour partie à un phénomène pour nous des plus important: dans bien des cas, au sein de la sous-caste (la fraction locale d'une caste), le vote fait l'objet d'une délibération. Rendant compte des élections de 1980, James Manor écrit à ce propos :

"Ces délibérations tendaient à être des activités de groupes, et les décisions concernant le vote se révélèrent avoir été prises collectivement et non pas individuellement dans un très grand nombre de cas. Une forte minorité de ces choix était très fortement inspirée, généralement de façon décisive, par des "leaders d'opinion" qui étaient des personnalités très différentes des puissants propriétaires fonciers qui recouraient jadis à l'intimidation pour imposer leurs vues aux villageois. Il s'agissait plutôt de personnes diplômées du secondaire, de l'université, et qui connaissaient le fonctionnement de l'administration et du système judiciaire. Ils avaient souvent aidé les villageois dans leurs relations avec une autorité supra-locale, d'une façon ou d'une autre, qu'il s'agisse de choses mineures comme remplir un formulaire, ou de cas plus sérieux comme défendre les intérêts de villageois impliqués dans des conflits ou obtenir pour eux une part des financements gouvernementaux ... Il s'agissait souvent d'enseignants, d'employés, de membres des associations bénévoles ou des coopératives, de membres ou d'employés des agences de développement ou, apparemment moins souvent, d'avocats de petites villes ${ }^{1}$.

Le vote apparaît ici à la fois comme un acte collectif et rationnel. L'influence extérieure n'est plus celle des dominants traditionnels, elle s'exprime plutôt par des conseils que le groupe sollicite pour voter au mieux de ses intérêts. L'attirail mis en cuvre par la commission électorale pour garantir un vote secret et raisonné paraît ici quelque peu décalé. S'il améliore la fiabilité des élections, c'est pour faire émerger non pas un électeur individuel et rationnel, mais un électeur collectif et rationnel : il a aidé, en effet, les basses castes à s'émanciper de la tutelle des dominants, et celles-ci sont assez fortes à présent - en raison, par ailleurs, de leur poids démographique et même économique - pour agir comme des groupes d'intérêt au moment du vote.

La sophistication des mesures prises par la commission électorale pour entourer le vote des meilleures garanties (en termes de secret, d'identification des électeurs, etc.) paraît aussi décalée par rapport à la dégradation des conditions élémentaires du vote.

1. J. Manor, «The electoral process amid awakening and decay: reflections on the Indian general elections of $1980 »$, dans P. Lyon, J. Manor (eds), Transfer and transformation, op. cit., p. 98. 


\section{Christophe Jaffrelot}

\section{Fraudes et violences}

L'enjeu principal réside en effet de plus en plus clairement dans la montée de la violence autour des bureaux de vote; car si l'acte électoral est entouré de toutes les garanties, il n'en est pas de même pour les urnes, voire les candidats, qui peuvent disparaître.

La fraude électorale la plus répandue consiste, pour des candidats ou des partis qui pressentent, le jour du scrutin, que le succès leur échappe, à louer les services d'un gang armé qui ira s'emparer d'une ou plusieurs urnes de la circonscription. En 1971, la commission électorale reconnaissait onze cas de ce type, dont huit au Bihar, réputé pour l'acuité de la violence politique ${ }^{1}$. En 1980 , près d'une soixantaine de bureaux de vote - dont vingt et un au Bihar, douze en Uttar Pradesh et dixneuf au Cachemire - furent le théâtre d'incidents comparables ou de tensions conduisant à annuler le scrutin ${ }^{2}$.

A ces pratiques s'ajoutent parfois des troubles autour des bureaux de vote: accrochages entre militants mais aussi agitations visant à suspendre le déroulement du scrutin. En 1989, ces violences ont fait une centaine de morts. Même si les cas restent rares, on observe aussi une multiplication des assassinats politiques visant les candidats eux-mêmes. En 1989, un des opposants au Congrès I, candidat à l'Assemblée législative d'Uttar Pradesh dans la circonscription dont Rajiv Gandhi disputait le siège au Parlement, reçut une balle dans le ventre.

En 1991, 460 compagnies de la Central Paramilitary Reserve Force furent déployées autour des bureaux de vote après que les violences accompagnant la campagne électorale eurent déjà fait soixante-quinze victimes dont cinquante au Bihar ${ }^{3}$. Dans certaines régions, l'acte de vote se déroule donc de plus en plus sous la menace et/ou sous la protection des armes.

L'Inde s'est dotée d'une procédure électorale propre à surmonter l'obstacle de l'analphabétisme; le système des symboles, perfectionné au fil des scrutins, permet à l'électeur d'exprimer sa préférence politique lors $\mathrm{du}$ vote dans de bonnes conditions d'indépendance, d'information et de secret. Cette procédure a contribué à simplifier et à institutionnaliser le système des partis, ce qui témoigne de l'importance des effets induits qu'un aménagement de l'acte de vote peut développer.

Le perfectionnement, voire le raffinement, de la procédure électorale, auxquels s'attache une commission électorale largement inspirée par les pratiques occidentales, semble toutefois en décalage par rapport aux réalités indiennes et aux enjeux présents : le vote n'apparaît comme acte

1. Report of the fifth general election, op. cit., p. 81 .

2. Report on the general elections to the House of the People, op. cit., p. 113-114.

3. National Mail, 8 mai 1991. 
individuel qu'à la marge de la société indienne, et le véritable défi réside plutôt dans la multiplication des fraudes violentes.

Le nouveau responsable de la commission électorale, Seshan, semble avoir adopté la bonne méthode en faisant revoter dans tous les bureaux de vote victimes de fraude, de façon plus systèmatique que par le passé. En 1991, on a revoté dans 2614 bureaux de vote (dont 1177 au Bihar et 876 en Uttar Pradesh, les deux Etats les plus travaillés par la violence politique). Mais une solution plus radicale consisterait à se dispenser des urnes. En 1982, la commission électorale avait envisagé d'installer un système de vote électronique. L'électeur n'avait plus qu'à appuyer sur le bouton situé en face du symbole et du nom du candidat de son choix, et son vote était automatiquement enregistré. L'expérience, menée lors de nombreuses élections partielles, fut abandonnée en raison du coût de cette installation ${ }^{1}$ puis, en 1989 , en raison de la défiance de l'opposition qui redoutait d'être trompée par le pouvoir. La commission électorale a cependant annoncé, après le scrutin de 1991, que cette réforme de l'acte de vote entrerait en vigueur dès les prochaines élections. Cette nouvelle réforme éviterait que l'on prenne d'assaut les bureaux de vote pour s'emparer des urnes, puisque celles-ci ne seraient plus utilisées.

Cette procédure permettrait aussi de remédier aux fraudes que favorise la lenteur de l'actuel système ${ }^{2}$. Certains partis envoient en effet des militants n'étant pas en âge de voter faire la queue dans les bureaux de vote où ils sont les plus vulnérables, de manière à faire perdre patience aux électeurs qui attendent leur tour ${ }^{3}$.

Christophe Jaffrelot est chargé de recherche au CNRS (Centre d'études et de recherches internationales, Fondation nationale des sciences politiques). Il est l'auteur de "Les modèles explicatifs de l'émergence des nations et du nationalisme; revue critique », dans Gil Delannoi, PierreAndré Taguieff (dir.). Théories du nationalisme, Paris, Kimé, 1991 et de "Les émeutes entre hindous et musulmans. Essai de hiérarchisation des facteurs culturels, économiques et politiques", Cultures et conflits, 5, printemps 1992. Il vient de publier Les nationalistes hindous. Idéologie, implantation et mobilisation, des années 1920 aux années 1990, Paris, Presses de la Fondation nationale des sciences politiques, 1993. Il travaille actuellement sur la démocratie en Inde et sur une anthologie d'Ambedkar, en collaboration avec Olivier Herrenschmidt (CERI, Maison des sciences de l'homme, 54 boulevard Raspail, 75007 Paris).

1. D. Butler, A. Lahiri, P. Roy, India decides, op. cit.,, p. 16.

2. Le recours à l'informatique ne résoudrait évidemment pas d'autres problèmes comme l'achat des voix des plus pauvres à coup de billets de banque ou de bouteilles d'alcool, la veille du scrutin. En 1991 , on estimait que les candidats à la Chambre basse investissaient en moyenne 1,5 à 2 millions de roupies dans leur campagne. Ces chiffres s'expliquent cependant aussi en partie par le coût des nouvelles technologies utilisées lors des campagnes électorales : un film vidéo revient à environ 300000 roupies, et la location mensuelle d'un véhicule équipé d'un magnétoscope et d'un écran pour le diffuser dans les rues, entre 60000 et 1,3 million de roupies (National Mail, 3 mai 1991).

3. Une première version de ce travail a fait l'objet d'une communication au Colloque organisé les 8 et 9 décembre 1992 par le Centre de recherches politiques de la Sorbonne et l'Association française de science politique. 
ANNEXE 1

Bulletin de vote utilisé lors des élections de 1957

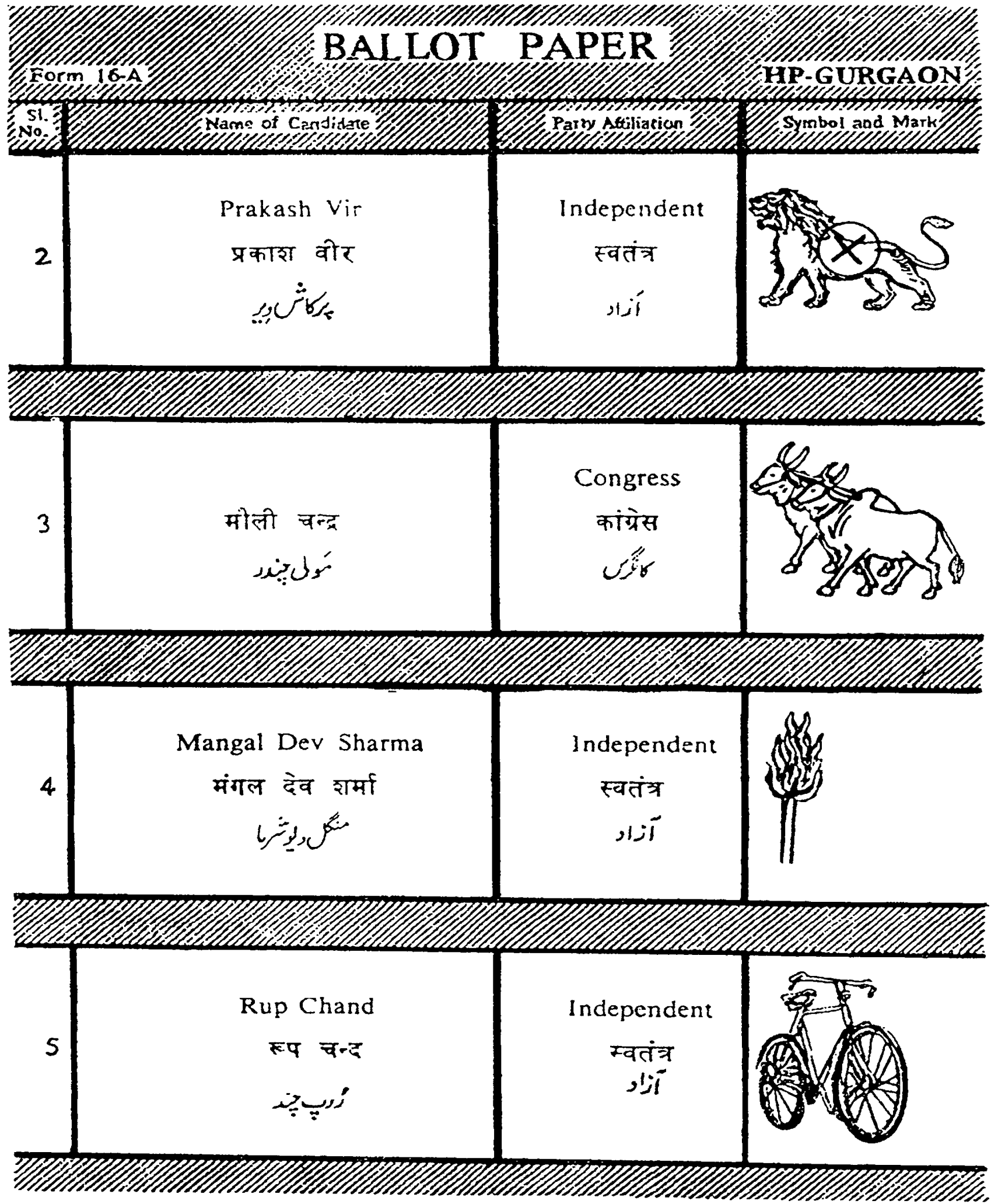

Source: Report on the second general elections in India, 1957, vol. 1, General, op. cit. 
ANNEXE 2

Symboles électoraux réservés par la commission électorale aux quatre partis nationaux et aux autres en 1957

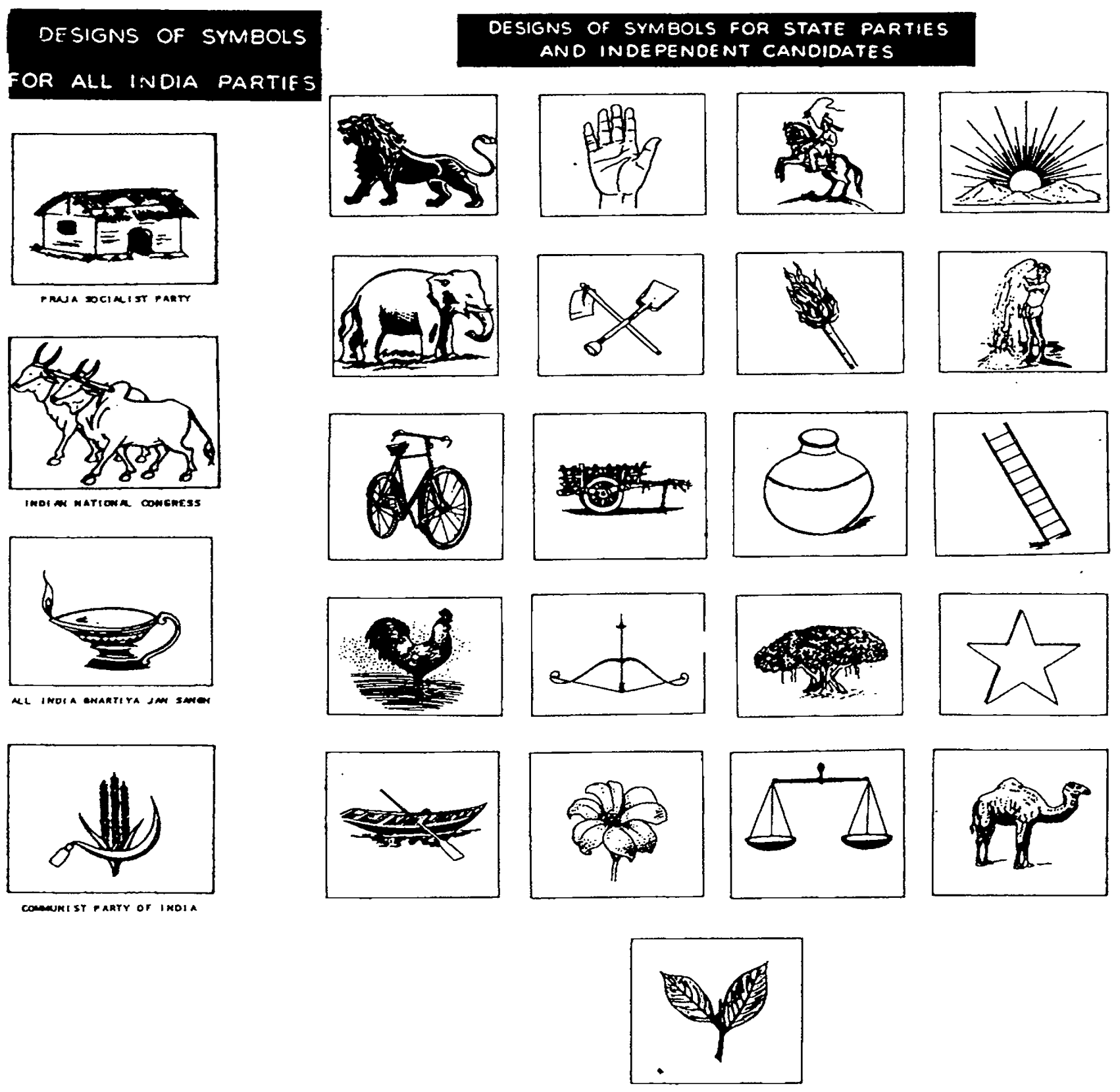

Source: Report on the second general elections in India, 1957, vol. 1, General, op. cit. 


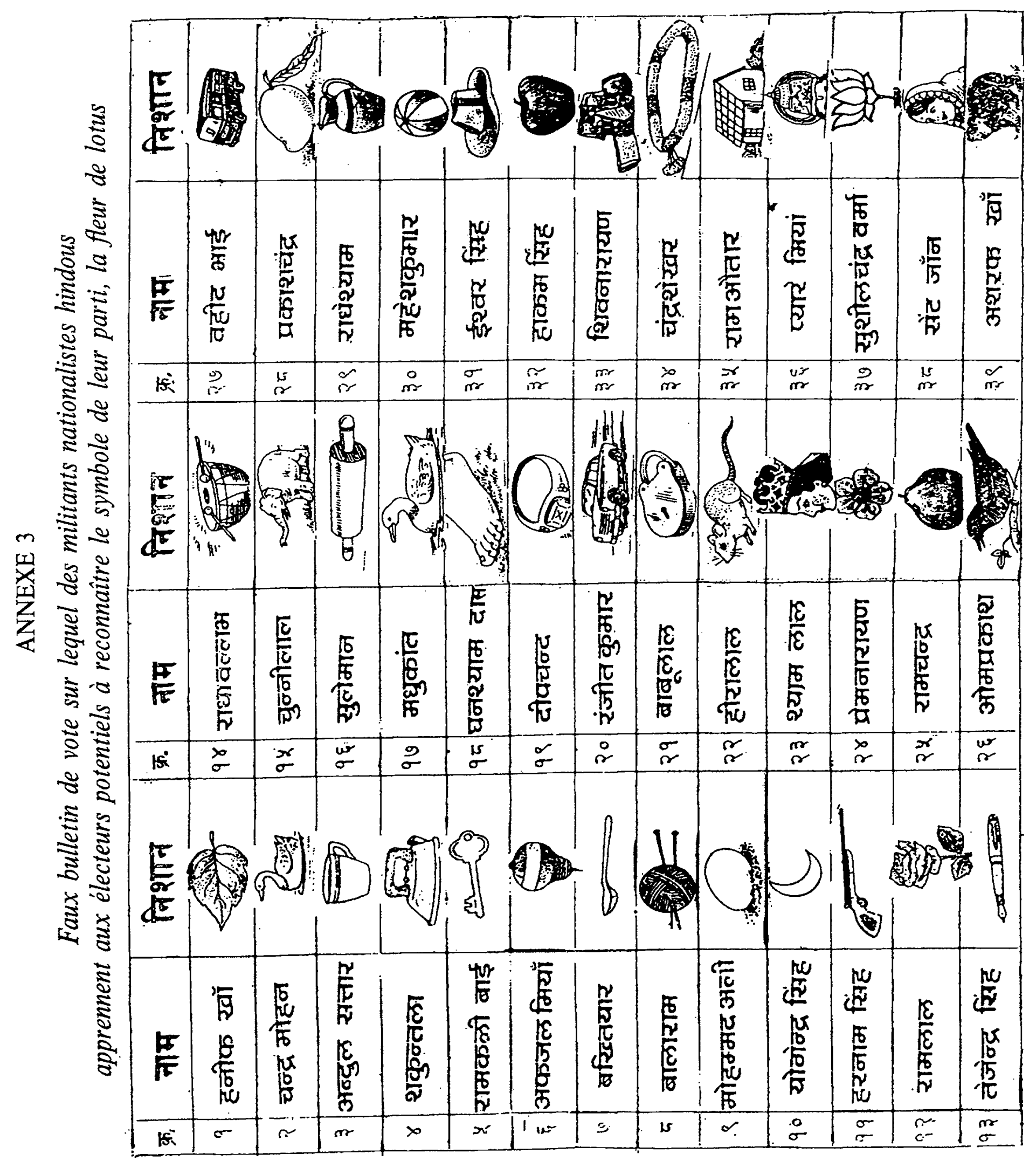

